\title{
University of California, Los Angeles, surgical time-out process: evolution, challenges, and future perspective
}

\author{
*Nancy Mclaughlin, M.D., Ph.D., ${ }^{1}$ Deborah Winograd, R.N., ${ }^{2}$ Hallie R. Chung, R.N., ${ }^{2}$ \\ Barbara Van de Wiele, M.D., ${ }^{3}$ And Neil A. Martin, M.D. ${ }^{1}$ \\ Departments of ${ }^{1}$ Neurosurgery and ${ }^{3}$ Anesthesiology, David Geffen School of Medicine; and ${ }^{2}$ Department of \\ Quality Management, Ronald Reagan UCLA Medical Center and Santa Monica UCLA Medical Center \& \\ Orthopaedic Hospital, University of California, Los Angeles, California
}

\begin{abstract}
Since the development of the WHO Safe Surgery Saves Lives initiative and Surgical Safety Checklist, numerous hospitals across the globe have adopted the use of a surgical checklist. The UCLA Health System developed its first extended Surgical Safety Checklist in 2008. Authors of the present paper describe how the time-out checklist used before skin incision was implemented and how it progressed to its current form. Compliance with the most recent version of the checklist has been closely monitored via documentation and observance audits. In addition, the surgical team's appreciation of the current time-out has been assessed. Cultural, practice, and human resource challenges are discussed, as are potential future avenues for innovations in the emerging field of the surgical checklist in neurosurgery.

(http://thejns.org/doi/abs/10.3171/2012.8.FOCUS12255)
\end{abstract}

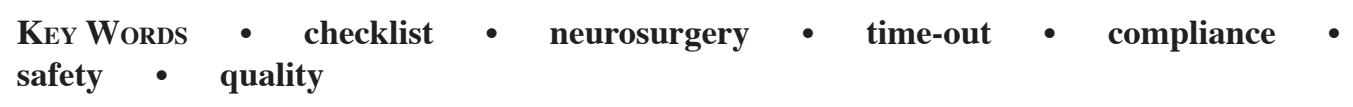

Cise HECKLISTS have been used in many industries to improve efficiency, accuracy, and safety. The first pilot's checklist was developed in the early 1930s as aircraft technology became more complex and the number of steps required by pilots for safe takeoff and landing increased. In the late 1990s Berenholtz and colleagues ${ }^{3}$ tested a safety checklist in the surgical intensive care unit at Johns Hopkins Hospital. They developed a 5-item checklist that was used before inserting central lines from January 1998 through December 2002. After 1 year of requiring completion of the checklist before all central line insertions, associated infections had dropped from $11 \%$ to $0 \%$ in the intensive care unit. Using statistical projections, the authors estimated that 43 infections were prevented, 8 lives were spared, and almost $\$ 2$ million in additional costs per year were saved. The success of the central line insertion checklist spread quickly, and today a similar checklist is used in most patient care settings across the nation. ${ }^{12,33,36} \mathrm{At}$ about the same time, Calland and colleagues ${ }^{4}$ performed a randomized controlled trial of procedural checklists to determine their capacity to increase the frequency of safetycritical behaviors during laparoscopic cholecystectomies. Cases were randomized from April 2001 to July 2002. Their study represents the first use of operative checklists in the US. No differences in patient outcome, case

\footnotetext{
Abbreviations used in this paper: SCIP = Surgical Care Improvement Project; UCLA = University of California, Los Angeles.

* Dr. McLaughlin and Ms. Winograd contributed equally to this work.
}

duration, and technical proficiency were noted between surgeons proceeding with a preprocedural briefing and checklist versus those proceeding in their standard fashion. Importantly, however, the study did show that surgical safety checklists increased the frequency of favorable team behavior such as team communication. ${ }^{4}$

Other health care checklists quickly followed in large hospitals and smaller practice settings across the globe. . $, 8,13,15,17,19$ A nursing pain management checklist was developed to ensure that a patient's pain level was assessed at least once every 4 hours. ${ }^{8}$ Following implementation of the checklist, untreated pain complaints decreased from $41 \%$ to $3 \%$. A checklist for patients on ventilators was created to ensure the head of the patient's bed was raised to $30^{\circ}, \mathrm{H} 2$ inhibitors were initiated, and frequent oral care was provided. ${ }^{2}$ After using the checklist, a $25 \%$ decrease in ventilator-associated pneumonia and 21 fewer deaths than in the prior year were noted.

The WHO was attentive to the safety and quality concerns in the massively growing number of surgeries performed annually around the world. In the US alone, studies suggested that almost half of all surgical complications in which patients were left disabled or dead were potentially preventable. ${ }^{7,34}$ Reports of operations mistakenly performed on the wrong extremity, or even on the wrong patient altogether, had surfaced. 6,14,21,28,32 Inadequately maintained medical equipment setting fire to patients, incorrect use of instruments due to improper staff training, miscommunication between medical teams, and preventable complications presented issues that needed immediate action. 
In January 2007, WHO officials and a panel of expert surgeons, anesthesiologists, nurses, and even patients from around the globe met at the WHO Geneva headquarters to discuss potential solutions. Shortly thereafter, the WHO Surgical Safety Checklist was created. ${ }^{24,46}$ In 2009 , the results of a multisite pilot study assessing the efficacy of the WHO surgical checklist was published in the New England Journal of Medicine. The authors observed a $4 \%$ decrease in surgery-related complications and a $0.7 \%$ reduction in in-hospital deaths. ${ }^{16}$ The proposed checklist was designed to be easy to use and applicable in many settings. Numerous hospitals, both small and large scale, have implemented the WHO checklist and continue to observe its positive impact. ${ }^{38,39,42,43}$ To date, few groups have reported their experience with a surgical checklist specifically for neurosurgery. ${ }^{9,18,22,25}$

\section{Implementing the First Surgical Safety Checklist at UCLA}

As of July 1, 2004, the UCLA Health System adopted the Universal Protocol created by The Joint Commission Board to address the continuing occurrence of wrongsite, wrong-procedure, and wrong-person surgery. ${ }^{30,35}$ The Universal Protocol includes preprocedure verification, site marking, and time-out. ${ }^{35}$ It was expanded to a series of requirements under The Joint Commission's 2003 and 2004 National Patient Safety Goals. The UCLA Health System had instituted measures to meet all related regulatory requirements before introducing its extended time-out.

Prior to 2008 a formal extended time-out did not exist in the UCLA Health System operating rooms. Although the hospitals and clinics had established system-wide policies and practice requirements to ensure readiness and safety before surgery, there was no standardized list of items that all team members would verify together before incision. Only the patient identity, operative site, and procedure to be performed were confirmed by an operating room nurse, an anesthesia provider, and a member of the surgical team. The surgeon would discuss the operative plan with the assistant residents and/or fellow. The anesthesia provider and surgeon would discuss management concerns. While these verifications and discussions occurred consistently before all surgical procedures, they did so at varying times and often without all team members present. Consequently, not all team members were aware of all the critical components. Communication between team members was fragmented, and readiness in the event of unplanned difficulty was not consistently optimized.

Realizing the opportunities for patient care improvement, UCLA followed the lead of other hospitals worldwide and developed the first extended Surgical Safety Checklist. While the extended Surgical Safety Checklist included verification before induction of anesthesia, time-out before skin incision, and debriefing before the patient leaves the room, in this paper we focus only on the time-out portion of the checklist. Modeling the WHO Surgical Safety Checklist, ${ }^{24,46}$ the UCLA checklist required the surgical team to stop all activity to time-out just before incision or start of the procedure. During the time-out, the entire surgical team would gather together to review critical components outlined on the checklist. The checklist was a final check of the patient's identity, procedure to be performed, and operative site and laterality. The checklist required that this verification be cross-referenced with the patient's signed consent, at least 2 physical identification sources including the patient arm band, and at least 2 patient identifiers (full name, date of birth, and medical record number). Like the WHO checklist, the UCLA checklist included a review of the patient's drug allergies, their need for blood products, and the availability of any required implants, devices, or equipment. The surgeon was required to review anticipated critical events, nonroutine steps, case duration, and anticipated blood loss with the entire team. Nurses were to discuss equipment readiness, and anesthesia providers were to review any anesthesia-specific concerns with the entire team. The time-out checklist was printed on brightly colored plastic cards that were placed in every operating room and procedure room. Nurses, technicians, anesthesia providers, surgeons, residents, fellows, and medical students were all trained on the time-out process that would occur before the start of any invasive procedure.

The concept of performing an official common pause and the introduction of a checklist represented major changes in culture and practice. ${ }^{10}$ Initially, the UCLA Health System found it challenging to integrate and apply the WHO-recommended practices before every invasive procedure. While the policies were successful at other institutions, the belief that UCLA would have similar benefits was not shared by all surgical team members. At the beginning, even in specialties in which compliance with the time-out was high, 3 observations were common: 1) not all team members actively participated, 2) some elements were rushed and not given enough discussion, and 3) the checklist itself was not referred to and some elements of the list were skipped or forgotten. Despite initial challenges and difficulties, active daily and weekly management of the change helped to ease the culture shock. Direct observation and feedback, operating room huddles, and reviewing the process of time and its importance during departmental meetings are a few examples of the reinforcement measures taken. Progressively, the time-out became a forum for discussing the operative plan as a cohesive team, allowing each person to understand the concerns of the other. Although critical elements and anticipated events were discussed between the attending surgeon and assisting resident and between the attending surgeon and anesthesiologist, inclusion of this discussion in the time-out with the entire operative team present was viewed as a significant improvement. ${ }^{39}$ Indeed, team workflow is particularly important during the critical steps, planned or unplanned, of an elective surgery and even more so in urgent surgeries. ${ }^{45}$ It is exactly in these situations that it is important for each team member to know exactly what to do. Surgeons, anesthesia providers, and nurses really began to appreciate the opportunity to clarify key elements and ask questions. Certain services embraced the practice enthusiastically and wanted to add supplemental components to further enhance the process.

\section{Evolution of the Time-Out Process}

As the time-out practice became commonplace 
across the organization, improvements to the process and additions to the checklist were underway. Indeed, the WHO encouraged modifications to fit with local practices. While UCLA's initial checklist included verification of numerous components, there was little emphasis on the importance of the concept of the pause. Truly stopping all activity to time-out and discuss concerns was to be an ongoing theme-not just an exercise for the beginning of the case. Thorough communication was emphasized, and team members were assured that everyone in the room had equal power to stop activity and voice concern if a safety issue arose. To stress each team member's responsibility of voicing concerns, a safety statement by the team leader was instituted. The safety statement was worded to stress that each member should feel at ease to voice questions or concerns at anytime during the procedure: "If anyone has any concerns now or at any point during the procedure, please voice them immediately."

In addition to the safety statement, team member introductions, inspired by the WHO checklist, were also incorporated. The surgeon was to state, "My name is John Smith and I am the attending surgeon"; and then the nurse, "My name is Jane Doe and I am the circulating nurse"; and so forth to include every single person in the room. Nursing students and medical students were also asked to introduce themselves and expected to voice any questions or concerns. Having team members introduce themselves by name and role would help to instill comfort and confidence to speak up in the operating room. It would also help create a team spirit and allow people who may have just met to be formally introduced to one another. These early additions to the checklist, which encouraged personalized interaction between members of the procedural team, were quickly incorporated into daily practice.

Previously in the UCLA Department of Neurosurgery, the surgeon or designee (that is, the resident or fellow) could conduct the time-out. Recently, however, it became policy for the attending surgeon to conduct the time-out in person. It is the attending surgeon's responsibility to set the tone for open communication and team spirit. He or she ensures that all team members, including the attending anesthesiologist, all neurosurgical and anesthesia residents and fellows, circulating nurses, operating room technicians, neuromonitoring technicians, and all other participants, are present and actively engaged in the time-out process. The time-out leader asks, "Can we do a time-out now?" This is the key phrase that lets all team members know that they must not only stop what they are doing but also gather around the patient so that everyone can be easily seen and heard. If some members persist in their activity, the time-out will not proceed beyond the introduction of all members gathered together. Note that the Department of Neurosurgery added a supplemental list of items to verify after completing the hospital's standardized checklist. Two important elements added to the extended time-out are reviews of critical imaging and use of lasers. Although not pertinent to all surgeries, these additions were found to be so valuable that they were later added to the Health System's standardized checklist.

In July 2011, the UCLA Health System released its most current version of the time-out checklist for use across the system (Fig. 1). This latest version included the 2 elements added by the Department of Neurosurgery, as well as all preincision SCIP measures. Since implementing this version of the checklist, compliance has been closely monitored via documentation and observance audits. In addition, the surgical team's appreciation of the most current version of the time-out was assessed, giving important feedback on culture change and avenues for improvement.

\section{Compliance With the Time-Out Process and Preincision Guidelines}

As mentioned previously, before 2008 no standardized surgical safety checklist was used in the UCLA Health System. After introducing the first extended version of the time-out, compliance with the time-out process and preincision guidelines was assessed specifically for the Department of Neurosurgery. The first quarter showed $77 \%$ compliance based on the documentation audit (Fig. 2). Within 6 months of applying the time-out procedure, compliance reached $96 \%$. After implementing the most recent version of the time-out process in July 2011, a documentation audit showed $100 \%$ compliance during 3 of the 4 quarters. Note that compliance was also assessed by someone watching the time-out process. A completed time-out implied that the checklist card was used, that all elements were reviewed, and that the entire team was present. An incomplete time-out implied that the checklist card was not used during the time-out, at least 1 element was skipped, or at least 1 team member was missing. The completion audit revealed that although the time-out process was performed in almost all cases in the last year, complete observance of the time-out process was noted in only $53 \%-62 \%$ of cases across the UCLA Health System. Importantly, this audit was performed across all surgical specialties performing procedures in the main operating rooms of both the Ronald Reagan UCLA Medical Center and the Santa Monica UCLA Medical Center and Orthopaedic Hospital. The next step is identifying why complete observance was not achieved in all cases. Is the standardized time-out perceived as inappropriate for some specialties or procedures? Are there any new cultural issues that must be addressed?

The addition of checklist items covering SCIP measures in the most recent version of the time-out process was an initiative to improve UCLA's compliance with SCIP measures. Originally developed by the Centers for Medicare \& Medicaid Services and the Centers for Disease Control and Prevention, the SCIP national partnership endeavored to improve the quality and safety of surgical care by reducing postoperative complications..$^{1,11,37}$ The introduction of preincision SCIP measures to the time-out process was intended to improve UCLA's compliance with these nationally mandated process measures (Fig. 3). Between 2008 and June 2011, administration of the correct prophylactic antibiotic per institutional guidelines varied from $75 \%$ to $98 \%$. After performing the current version of the time-out process, this rate reached $100 \%$ and was maintained for the entire year. Admin- 


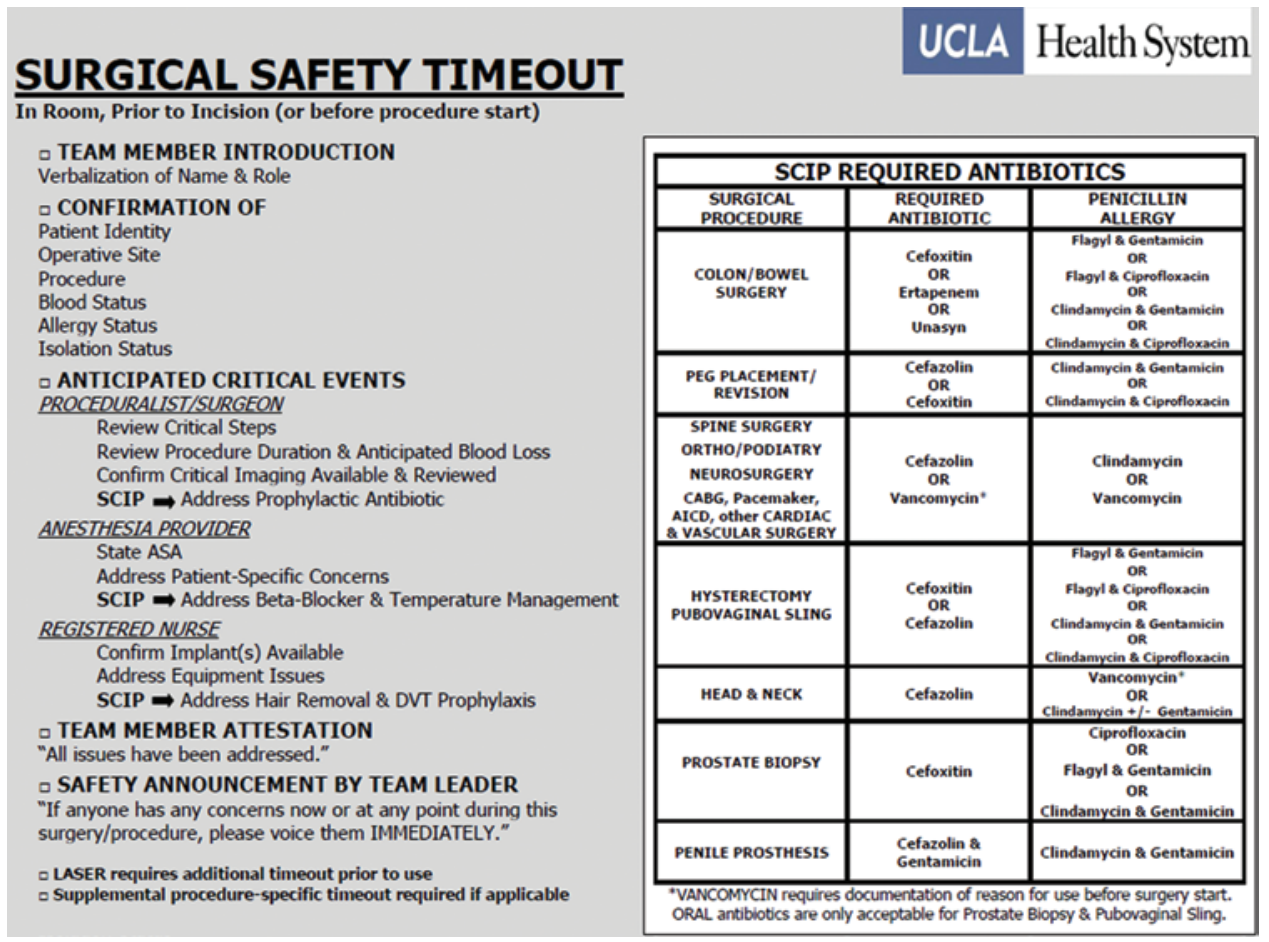

FIG. 1. The current extended Surgical Safety Time-Out checklist used in the UCLA Health System operating rooms. AICD = automatic implantable cardioverter defibrillator; $A S A=$ aspirin; $C A B G=$ coronary artery bypass graft; DVT = deep venous thrombosis; $P E G$ = percutaneous endoscopic gastrostomy.

istration of a beta-blocker in the perioperative period for appropriate patients ranged from $75 \%$ to $97 \%$ after instituting the first time-out but reached $100 \%$ and was maintained for the entire year after applying the current time-out. Compliance with intraoperative deep vein thrombosis prophylaxis (compression devices applied before incision) varied from $81 \%$ to $98 \%$ between 2008 and June 2011 but reached 100\% since July 2011. Intraoperative temperature management (application of forced air warming if the body temperature was below $36^{\circ} \mathrm{C}$ ) ranged from $98 \%$ to $100 \%$ after implementation of the initial time-out. Since enforcing the current time-out, the rate reached $100 \%$ and was maintained. Appropriate hair removal methods (clippers only, no razors) were met in $91 \%-100 \%$ of cases prior to June 2011. Thereafter, com- pliance reached $100 \%$ and was maintained. Thus, there was across-the-board improvement in compliance with SCIP guidelines after their incorporation into the checklist format of the time-out process.

\section{Appreciation of the Time-Out Process by the Team}

One year after implementing the most recent time-out process, members of the surgical team involved in neurosurgical procedures in the main operating theater of the Ronald Reagan UCLA Medical Center were asked to respond anonymously to a survey. This survey was intended to poll the team regarding its opinions on the time-out process. Responses were categorized as strongly agree, agree, disagree, strongly disagree. The respondents were also

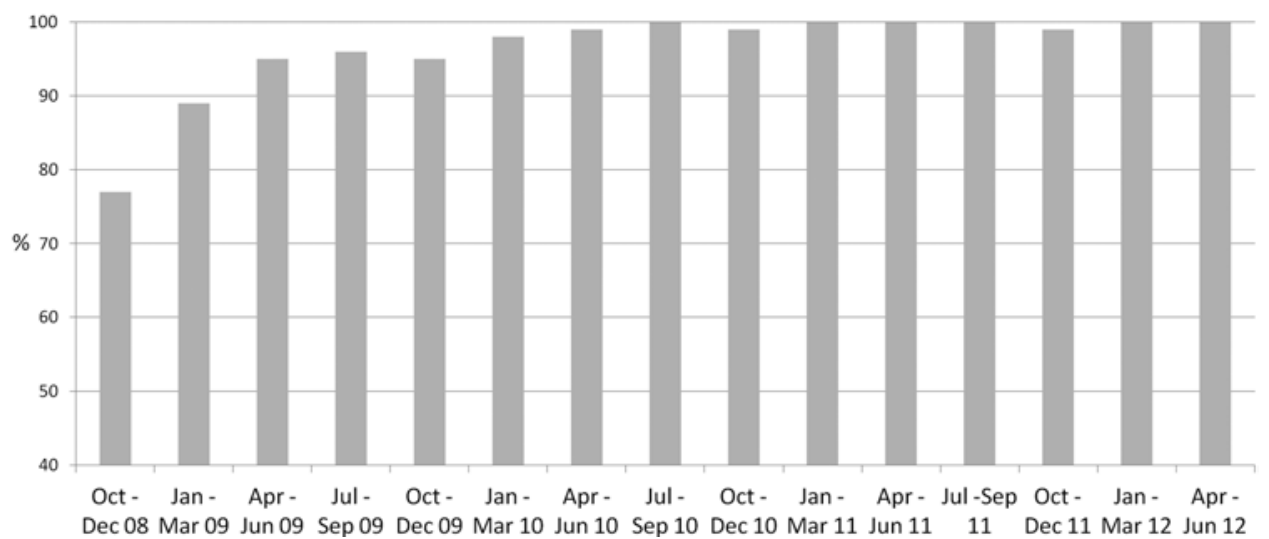

FiG. 2. Bar graph showing documentation audit results in the Department of Neurosurgery since implementation of the extended time-out. The y axis measures the compliance percentage. 


\section{The UCLA surgical time-out process}

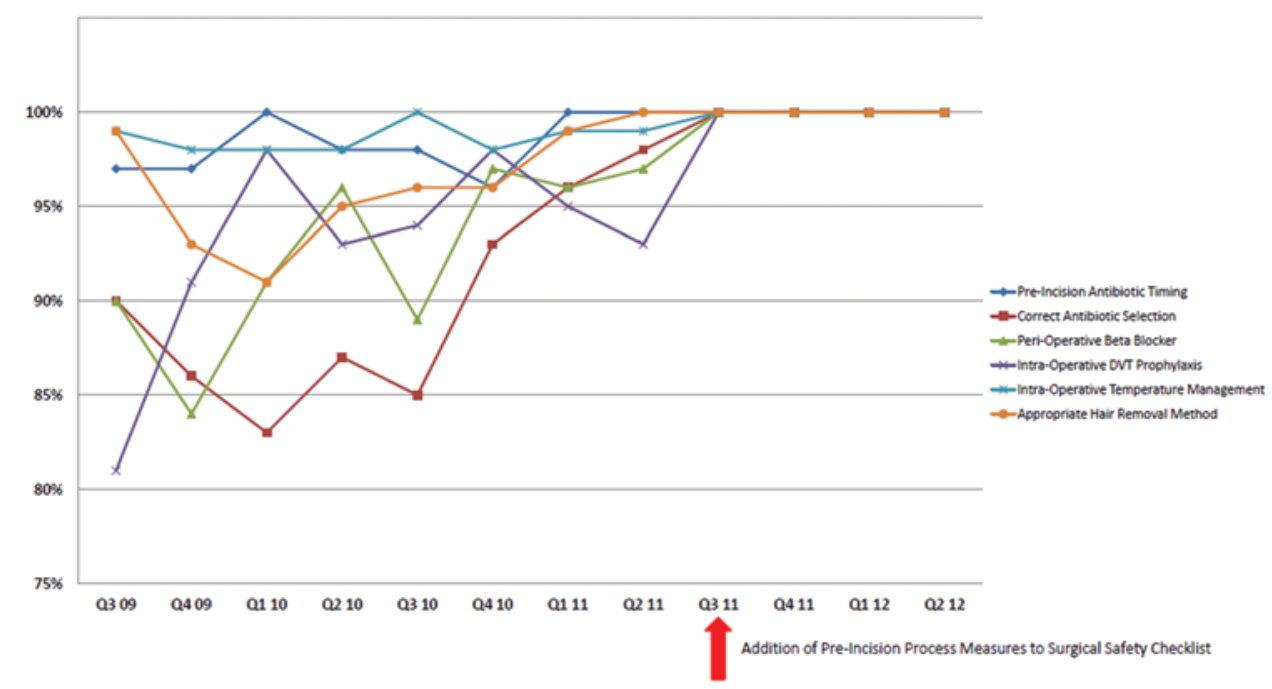

FIG. 3. Graph showing compliance with preincision process measures in the Department of Neurosurgery. $Q=$ quarter.

encouraged to note comments in a blank space reserved for this purpose. Ninety-three of the 128 members of the surgical team, including neurosurgical residents and/or fellows, neurosurgeons, anesthesia residents, anesthesiologists, operating room nurses, scrub technicians, and neuromonitoring technicians, completed the survey. Overall, $98.9 \%$ of respondents believed that performing a time-out prior to procedure start (incision) improves patient safety; $80.6 \%$ strongly agreed (Fig. 4A). The majority of respondents $(97.8 \%)$ believed that team member introductions helped to promote a team spirit during the case. Although $80.6 \%$ of the respondents strongly agreed with this statement, open comments stressed the importance of clearly pronouncing names (Fig. 4B). This step may be viewed as redundant when the same team is working together regularly. However, given the resident rotations in neurosurgery and anesthesia, the frequent training of new operating room personnel, and observation by medical students, the team member introductions were believed to be essential. Although $93.5 \%$ of respondents believed that performing a time-out helped to ensure all team members were comfortable to voice safety concerns throughout the case, only $59.1 \%$ of the respondents strongly agreed (Fig. 4C). Open comments stressed that the existence of a safety statement at the end of the UCLA time-out is an important reminder that all members should voice their concerns. However, it is crucial that the attending surgeon sets the tone favoring communication during the case. The time-out is a platform for the attending to inspire respect among all members of the team and make everyone feel that they are equally part of the team. All of the respondents believed that the attending surgeon's review of anticipated critical elements was helpful to their role during the case; $88.2 \%$ strongly agreed. A total of $97.1 \%$ of respondents believed that they learn something new about the patient and/or the procedure during the time-out that helps them ensure patient safety during the case; $69.1 \%$ strongly agreed. All respondents noted that they would want a time-out to be performed prior to the start (incision) of their own surgery if they were the patient. Overall, this survey showed that the team favorably views the use of the time-out.

\section{Challenges}

Since the institution of the first extended version of the time-out process, multiple challenges have surfaced. ${ }^{10,43,44}$ Some have been successfully tackled, whereas others are ongoing concerns.

\section{Cultural Change}

The cultural change was a challenge during implementation of the first version of the time-out. Surgeons have historically identified themselves as individual craftsman. Accepting that they are members of a team did require a culture change. ${ }^{40}$ The time-out process stimulates communication among all team members and encourages members to speak up during the time-out and at any time during the procedure. ${ }^{20}$ Some physicians were not used to being challenged, and some of the team members were not used to expressing their concerns. Surgeons value their clinical autonomy and were reluctant to proceed with a standardized preincision process. It was important to stress that the time-out was an accumulation of standardized prompts to stimulate discussion..$^{20,27}$ The checklist cannot encompass every situation, and the discretion and insight of the surgeon are essential.

\section{Practice Change}

The time-out differentiated itself from the formal preprocedural process of verifying the correct patient, procedure, and site. Gathering the entire team and making sure that everyone stopped activity to concentrate on the time-out process was a practice change and, in fact, was viewed by some as in an interruption of the workflow. ${ }^{10}$ Moreover, in the beginning, going through elements of the time-out took some time, since the team members were not acquainted with the process. Time spent in the operating theater is definitely costly. ${ }^{23,31}$ Realistically, the time-out takes approximately 3-5 minutes to complete. ${ }^{20}$ However, minor problems, distraction, and equipment issues were also associated with increased time in the operating room and decreased operative performance..$^{5,26}$ With time, the entire team embraced the fact that it was 
A Generally speaking, do you feel that performing a timeout prior to procedure start (incision) improves patient safety?

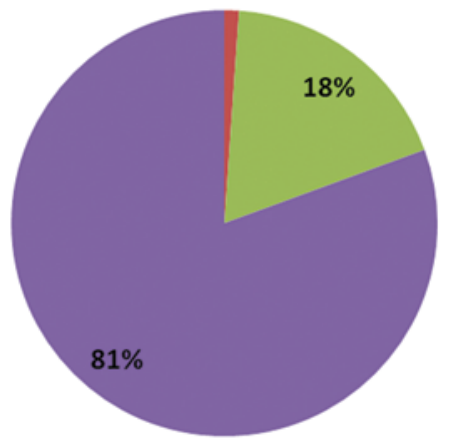

n Strongly Disagree: 0/93

Disagree: $1 / 93$

Agree: $17 / 93$

- Strongly Agree: 75/93

Do you feel that the team member introductions (each person announcing their name and role) helps to promote a team mentality during the case?

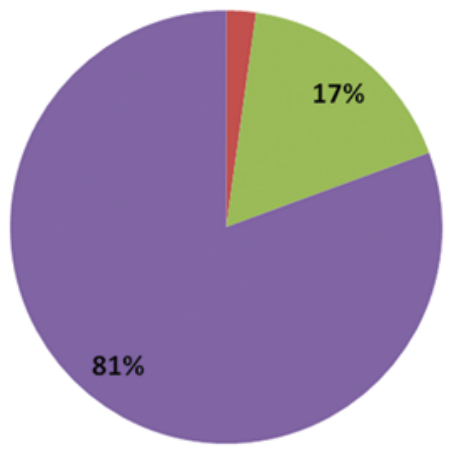

n Strongly Disagree: $0 / 93$

Disagree: $2 / 93$

agree: $16 / 93$

- Strongly Agree: $75 / 93$

C Do you feel that performing a timeout and team member introductions helps to ensure ALL team members are comfortable to voice safety concerns throughout the case?

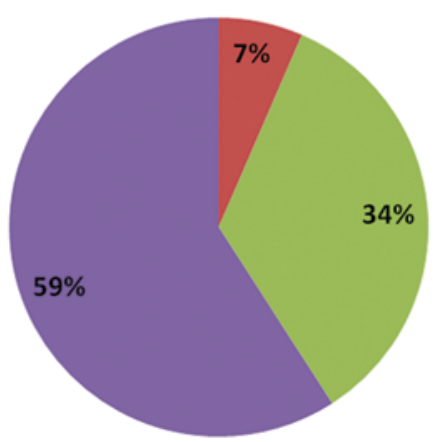

- Strongly Disagree: 0/93

Disagree: $6 / 93$

nagree: $32 / 93$

atrongly Agree: 55/93

FIG. 4. Charts showing results of the time-out appreciation survey performed in the Department of Neurosurgery.

important to take a few minutes at the beginning of a procedure to prevent losing time during the case and potentially during a critical step. The evolving safety culture supported these practice changes. Leadership endorsement early in the implementation process and throughout its entire progress has been instrumental. ${ }^{38}$

\section{Standardization of the Time-Out}

Using a standardized tool for all surgical procedures, whatever the duration, location, or complexity, is a challenge. A reasonable question arises: should the time-out be subspecialty-specific, surgeon-specific, and locationspecific (operating room, emergency department, or clinic)? The advantage of a standardized surgical checklist is that all members of the team know exactly the contents of the time-out, since it is the same from specialty to specialty. Given that the time-out process represents a template setting the stage for discussion prior to surgery, the team leader should be encouraged to discuss additional important elements for a specific patient or surgery during the time-out. The UCLA Health System has adopted the time-out checklist not only in the main operating rooms but also prior to invasive procedures performed in all patient care areas.

\section{Human Resource Obstacles}

Although the time-out is performed at the beginning of the procedure, staff turnover does occur during the case. Information transfers are made between nurses, scrub technicians, neuromonitoring technicians, residents, and 
anesthesiologists. Transferring information introduces the risk of failing to discuss or passing along the wrong information. Of course, maintaining the integrity of the team for as long as possible is preferred. However, this raises the following question: do all team members that will be involved in the case need to be present at the time-out? Realistically, this is not feasible. To remedy this, some have recommended creating service-specific operating room teams. Thus, even if a member of this team was not present at the initial time-out, he or she would be familiar with the cases and have knowledge of the critical elements usually discussed at the time-out. Turnover of team members is a challenge, emphasizing the need for frequent new training sessions, coaching sessions, and updates. ${ }^{29}$

\section{Future Perspectives}

As the team becomes used to performing the time-out, it becomes susceptible to checklist fatigue, in which the users become too familiar with the process and may go through the elements too quickly or not as thoroughly as before. Keeping the time-out process simple with a limited number of elements helps to prevent checklist fatigue. Innovative approaches from the time-out committee are also essential to maintain the interest of team members. As noted, the time-out process sets the tone for patient safety in the operating room. Each team member must remain vigilant throughout the case and avoid complacency toward patient safety. Training and continuous updates are essential to reeducate and stimulate team members.

In the era of informatics, contemplating the use of a smart checklist is not so far away. As in other institutions, the time-out card has been converted from paper to an electronic format. ${ }^{38}$ Since instituting the current version of the time-out, the neurosurgery department has been using an electronic format projected on digital screens in the operating theater. In the future, the surgeon might enter responses using a mobile tablet with screen touch technology that projects to screens. Using an interactive platform, the team would go through a series of essential elements. A specific response to an element might prompt a drop-down window to stimulate discussion on additional elements. This could prevent over-standardization and lengthening of the surgical checklist and time-out process. In addition, the data entered could be simultaneously compiled and immediately available for review.

\section{Conclusions}

Almost 4 years have passed since the introduction of the first extended Surgical Safety Checklist at UCLA. Striving to improve overall patient care, the UCLA Health System did not step back when facing culture and practice challenges. Regular evaluations and feedback have been instrumental in molding the time-out process into its current form and current level of appreciation by the team. The safety benefits of the surgical checklist rely on a structured and standardized communication protocol. ${ }^{41}$ The time-out process, including the pause, team member introductions, review of the checklist, and safety statement, is probably at the heart of the benefits in communication, collaboration, safety attitudes, and team-building experi- ence. Surveying the surgical team has shed light on features that require improvement and/or development, such as the debriefing process. Having joined the Best Practices for Better Care national collaborative in 2012, the UCLA Health System will continue to ensure safer surgeries. In addition to documenting the use of time-out checklists and reporting compliance, it will be important to demonstrate sound understanding of the process by all team members.

\section{Disclosure}

The authors report no conflict of interest concerning the materials or methods used in this study or the findings specified in this paper.

Author contributions to the study and manuscript preparation include the following. Conception and design: Martin, McLaughlin, Winograd. Acquisition of data: McLaughlin, Winograd, Dash. Analysis and interpretation of data: Martin, McLaughlin, Winograd, Van de Wiele. Drafting the article: McLaughlin. Critically revising the article: Martin, McLaughlin, Winograd, Dash, Van de Wiele. Reviewed submitted version of manuscript: all authors. Study supervision: Martin.

\section{References}

1. Berenguer CM, Ochsner MG Jr, Lord SA, Senkowski CK: Improving surgical site infections: using National Surgical Quality Improvement Program data to institute Surgical Care Improvement Project protocols in improving surgical outcomes. J Am Coll Surg 210:737-743, 2010

2. Berenholtz SM, Milanovich S, Faircloth A, Prow DT, Earsing $\mathrm{K}$, Lipsett $\mathrm{P}$, et al: Improving care for the ventilated patient. Jt Comm J Qual Saf 30:195-204, 2004

3. Berenholtz SM, Pronovost PJ, Lipsett PA, Hobson D, Earsing K, Farley JE, et al: Eliminating catheter-related bloodstream infections in the intensive care unit. Crit Care Med 32:20142020, 2004

4. Calland JF, Turrentine FE, Guerlain S, Bovbjerg V, Poole GR, Lebeau K, et al: The surgical safety checklist: lessons learned during implementation. Am Surg 77:1131-1137, 2011

5. Catchpole KR, Giddings AE, Wilkinson M, Hirst G, Dale T, de Leval MR: Improving patient safety by identifying latent failures in successful operations. Surgery 142:102-110, 2007

6. Cohen FL, Mendelsohn D, Bernstein M: Wrong-site craniotomy: analysis of 35 cases and systems for prevention. Clinical article. J Neurosurg 113:461-473, 2010

7. de Vries EN, Ramrattan MA, Smorenburg SM, Gouma DJ, Boermeester MA: The incidence and nature of in-hospital adverse events: a systematic review. Qual Saf Health Care 17: 216-223, 2008

8. Erdek MA, Pronovost PJ: Improving assessment and treatment of pain in the critically ill. Int J Qual Health Care 16: 59-64, 2004

9. Fargen KM, Velat GJ, Lawson MF, Firment CS, Mocco J, Hoh BL: Enhanced staff communication and reduced near-miss errors with a neurointerventional procedural checklist. J Neurointerv Surg [epub ahead of print], 2012

10. Fourcade A, Blache JL, Grenier C, Bourgain JL, Minvielle E: Barriers to staff adoption of a surgical safety checklist. BMJ Qual Saf 21:191-197, 2012

11. Fry DE: Surgical site infections and the surgical care improvement project (SCIP): evolution of national quality measures. Surg Infect (Larchmt) 9:579-584, 2008

12. Galpern D, Guerrero A, Tu A, Fahoum B, Wise L: Effectiveness of a central line bundle campaign on line-associated infections in the intensive care unit. Surgery 144:492-495, 2008

13. Gawande A: The Checklist Manifesto: How to Get Things Right. New York: Metropolitan Books, 2010

14. Giles SJ, Rhodes P, Clements G, Cook GA, Hayton R, Max- 
well MJ, et al: Experience of wrong site surgery and surgical marking practices among clinicians in the UK. Qual Saf Health Care 15:363-368, 2006

15. Hanna B, Jarman H, Savage S, Layton K: The early detection of postpartum depression: midwives and nurses trial a checklist. J Obstet Gynecol Neonatal Nurs 33:191-197, 2004

16. Haynes AB, Weiser TG, Berry WR, Lipsitz SR, Breizat AH, Dellinger EP, et al: A surgical safety checklist to reduce morbidity and mortality in a global population. N Engl J Med 360: 491-499, 2009

17. Hui-Chi H: A checklist for assessing the risk of falls among the elderly. J Nurs Res 12:131-142, 2004

18. Kramer DR, Halpern CH, Connolly PJ, Jaggi JL, Baltuch GH: Error reduction with routine checklist use during deep brain stimulation surgery. Stereotact Funct Neurosurg 90:255-259, 2012

19. Kruijver IP, Garssen B, Visser AP, Kuiper AJ: Signalising psychosocial problems in cancer care: the structural use of a short psychosocial checklist during medical or nursing visits. Patient Educ Couns 62:163-177, 2006

20. Lingard L, Espin S, Rubin B, Whyte S, Colmenares M, Baker GR, et al: Getting teams to talk: development and pilot implementation of a checklist to promote interprofessional communication in the OR. Qual Saf Health Care 14:340-346, 2005

21. Loft ES, Marcus DM: Wrong-site eye surgery. Ophthalmic Surg Lasers Imaging 35:182-184, 2004

22. Lyons MK: Eight-year experience with a neurosurgical checklist. Am J Med Qual 25:285-288, 2010

23. Macario A: What does one minute of operating room time cost? J Clin Anesth 22:233-236, 2010

24. Mahajan RP: The WHO surgical checklist. Best Pract Res Clin Anaesthesiol 25:161-168, 2011

25. McConnell DJ, Fargen KM, Mocco J: Surgical checklists: a detailed review of their emergence, development, and relevance to neurosurgical practice. Surg Neurol Int 3:2, 2012

26. Mishra A, Catchpole K, Dale T, McCulloch P: The influence of non-technical performance on technical outcome in laparoscopic cholecystectomy. Surg Endosc 22:68-73, 2008

27. Nagpal K, Vats A, Lamb B, Ashrafian H, Sevdalis N, Vincent $\mathrm{C}$, et al: Information transfer and communication in surgery: a systematic review. Ann Surg 252:225-239, 2010

28. Neily J, Mills PD, Eldridge N, Dunn EJ, Samples C, Turner JR, et al: Incorrect surgical procedures within and outside of the operating room. Arch Surg 144:1028-1034, 2009

29. Neily J, Mills PD, Young-Xu Y, Carney BT, West P, Berger $\mathrm{DH}$, et al: Association between implementation of a medical team training program and surgical mortality. JAMA 304:1693-1700, 2010

30. Norton E: Implementing the universal protocol hospital-wide. AORN J 85:1187-1197, 2007

31. Park KW, Dickerson C: Can efficient supply management in the operating room save millions? Curr Opin Anaesthesiol 22:242-248, 2009

32. Perlow DL, Perlow SM: Incidence of wrong-site surgery among hand surgeons. J Bone Joint Surg Am 85-A:1849, 2003 (Letter)

33. Pronovost P, Needham D, Berenholtz S, Sinopoli D, Chu H, Cosgrove S, et al: An intervention to decrease catheter-related bloodstream infections in the ICU. N Engl J Med 355:27252732, 2006
34. Rothschild JM, Landrigan CP, Cronin JW, Kaushal R, Lockley SW, Burdick E, et al: The Critical Care Safety Study: the incidence and nature of adverse events and serious medical errors in intensive care. Crit Care Med 33:1694-1700, 2005

35. Saufl NM: Universal protocol for preventing wrong site, wrong procedure, wrong person surgery. J Perianesth Nurs 19:348-351, 2004

36. Schulman J, Stricof R, Stevens TP, Horgan M, Gase K, Holzman IR, et al: Statewide NICU central-line-associated bloodstream infection rates decline after bundles and checklists. Pediatrics 127:436-444, 2011

37. Stulberg JJ, Delaney CP, Neuhauser DV, Aron DC, Fu P, Koroukian SM: Adherence to surgical care improvement project measures and the association with postoperative infections. JAMA 303:2479-2485, 2010

38. Styer KA, Ashley SW, Schmidt I, Zive EM, Eappen S: Implementing the World Health Organization surgical safety checklist: a model for future perioperative initiatives. AORN J 94:590-598, 2011

39. Takala RS, Pauniaho SL, Kotkansalo A, Helmiö P, Blomgren $\mathrm{K}$, Helminen M, et al: A pilot study of the implementation of WHO surgical checklist in Finland: improvements in activities and communication. Acta Anaesthesiol Scand 55:12061214,2011

40. Taylor B, Slater A, Reznick R: The surgical safety checklist effects are sustained, and team culture is strengthened. Surgeon 8:1-4, 2010

41. Thomas EJ, Sherwood GD, Helmreich RL: Lessons from aviation: teamwork to improve patient safety. Nurs Econ 21:241243,2003

42. van Klei WA, Hoff RG, van Aarnhem EE, Simmermacher RK, Regli LP, Kappen TH, et al: Effects of the introduction of the WHO "Surgical Safety Checklist" on in-hospital mortality: a cohort study. Ann Surg 255:44-49, 2012

43. Vats A, Vincent CA, Nagpal K, Davies RW, Darzi A, Moorthy $\mathrm{K}$ : Practical challenges of introducing WHO surgical checklist: UK pilot experience. BMJ 340:b5433, 2010

44. Walker IA, Reshamwalla S, Wilson IH: Surgical safety checklists: do they improve outcomes? Br J Anaesth 109:47-54, 2012

45. Weiser TG, Haynes AB, Dziekan G, Berry WR, Lipsitz SR, Gawande AA: Effect of a 19-item surgical safety checklist during urgent operations in a global patient population. Ann Surg 251:976-980, 2010

46. Weiser TG, Haynes AB, Lashoher A, Dziekan G, Boorman DJ, Berry WR, et al: Perspectives in quality: designing the WHO Surgical Safety Checklist. Int J Qual Health Care 22: $365-370,2010$

Manuscript submitted July 13, 2012.

Accepted August 31, 2012.

Please include this information when citing this paper: DOI: 10.3171/2012.8.FOCUS12255.

Address correspondence to: Neil A. Martin, M.D., Department of Neurosurgery, David Geffen School of Medicine at UCLA, Ronald Reagan UCLA Medical Center, 757 Westwood Plaza, Suite 6236, Los Angeles, California 90095-7436. email: neilmartin@mednet. ucla.edu. 